\title{
The Role of Wnt/Beta-Catenin Pathway, SATB1 and SATB2 in Endometrial Cancer Patients
}

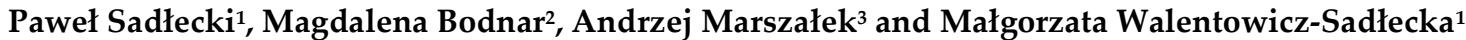

${ }^{1}$ Department of Obstetrics, Gynecology and Oncologic Gynecology, Collegium Medicum in Bydgoszcz, Nicolaus Copernicus University in Torun, Bydgoszcz, Poland.

${ }^{2}$ Department of Clinical Pathomorphology, Collegium Medicum in Bydgoszcz, Nicolaus Copernicus University in Torun, Bydgoszcz, Poland.

${ }^{3}$ Chair of Oncologic Pathology and Prophylaxis Poznan University of Medical Sciences \& Greater Poland Cancer Center Poznan, Poland.

* Correspondence: author: Pawel Sadlecki, sadleckip@gmail.com ; Department of Obstetrics and Gynecology, L. Rydygier Collegium Medicum in Bydgoszcz, Nicolaus Copernicus University; ul. Ujejskiego 75, Bydgoszcz 85-168, Poland Tel.: +48 693529294; Fax: +48-523655245.

ABSTRACT: Wnt/ $\beta$-catenin signaling pathway plays an established role in various diseases and is considered a hallmark of endometrial cancer (EC). Special AT-rich sequence-binding protein 1 and 2 (SATB1 and SATB2) are nuclear matrix-associated proteins involved in chromatin remodeling and regulation of gene expression. SATB1 promotes the progression of numerous types of cancers, whereas SATB2 acts as a tumor suppressor. Despite a recent progress in our knowledge about EC, the exact mechanisms that control their proliferation and metastatic potential still remain unknown.

The aim of our study was to investigate the association between Wnt3A, $\beta$ catenin, SATB1 and SATB2 protein level and the clinicopathological features of EC patients.

92 EC patients, aged 37-84, were enrolled to our study. The immunoexpression of WNT3A was found in specimens from all EC patients, $\beta$-catenin was expressed in $97 \%$ of the cases, SATB1 in $87 \%$. The significant association between Wnt3a expression and tumor grade was found; moreover mean IRS for Wnt3a turned out to be significantly lower in high-grade tumors than in low-grade malignancies $(p=0.038)$. In turn, immunoexpression of $\beta$-catenin varied significantly across FIGO stages and was associated with the presence of lymph node metastases. Mean IRS for $\beta$-catenin in patients with lymph node metastases was significantly lower than in those without $(p=0.028)$. The Kaplan-Meier analyses demonstrated a stepwise impairment of cancer overall survival with increasing SATB1 expression.

In conclusion, both $\mathrm{Wnt} / \beta$-catenin signaling pathway and SATB1 contribute to progression of EC. Downregulation of $\beta$-catenin may predispose to lymphatic spread of EC. In turn, downregulation of Wnt3a seems to be characteristic for high-grade tumors, but probably does not play a role in formation of lymph node metastases. The important role of SATB1 as a predictor of poor survival and could be helpful in establishing a more accurate prognosis in endometrial cancer patients.

KEYWORDS: endometrial cancer; tumor microenvironment; Wnt/ $\beta$-catenin pathway; SATB1; SATB2 


\section{INTRODUCTION}

Tumor microenvironment plays an important role at each step of cancer development. Cancer cells constantly interact with extracellular matrix (ECM), fibroblasts, endothelial and inflammatory cells. Conditions that promote local or systemic spread of cancer cells are vital albeit still relatively unknown clinical issue. Tumor growth and metastatic potential are associated with constant interaction between cancer cells and their microenvironment ${ }^{1}$.

Endometrial cancer (EC) is the sixth most prevalent female malignancy worldwide, with approximately 58,3 new cases per 100,000 and 24,4 deaths per 100,000 recorded annually. EC is also the most common gynecological cancer in Europe and United States ${ }^{2}$. A total of 5,706 new EC cases have been diagnosed in Poland in 2013, which makes this malignancy the third most common among Polish women, after breast and lung cancer [Polish National Cancer Registry]. EC grows slowly and therefore, has relatively good prognosis. Furthermore, due to early clinical manifestation, this malignancy is typically diagnosed at less advanced stages. As a result, 5-year overall survival (OS) rates in EC are high, more than $80 \%$ for all stages and more than $90 \%$ for stage $\mathrm{I}^{3}$. However, in a certain subset of patients prognosis is less favorable; such women may benefit from more aggressive anticancer treatments, namely adjuvant chemo- or radiotherapy. This more aggressive phenotype of EC is characterized by local infiltration and lymphatic spread of cancer cells. The risk of local and systemic spread determines the choice between various therapeutic strategies in EC: surgery with or without adjuvant radiochemotherapy, or radiochemotherapy alone. This stimulated research on prognostic markers that would accurately identify patients who require more aggressive treatment due to increased risk of EC spread, even at the expense of the quality of life deterioration, and those who may benefit from the surgery alone. Optimally, such markers should be also reliable predictors of recurrence and mortality in EC.

Despite a recent progress in our knowledge about cancer cells, the exact mechanisms that control their proliferation and metastatic potential still remain unknown. Wnt/ $\beta$-catenin signaling pathway plays an established role in various diseases and is considered a hallmark of colorectal tumorigenesis. This pathway controls crucial processes involved in embryogenesis and self-renewal of stem cells, such as proliferation, differentiation and migration ${ }^{4,5}$. Human genome includes 19 WNT genes that encode Wnt glycoproteins. Each Wnt protein has molecular weight of ca $40 \mathrm{kDa}$ and is composed of cysteine-rich aminoacids $s^{6}$. The first described protein from the WNT family is Wnt3a,

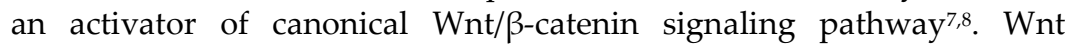
proteins bind to cell surface receptors from the Frizzled family (Fz); this process may require involvement of a co-receptor, lipoprotein receptorrelated protein (LRP) ${ }^{6}$.

Wnt proteins transduce signal via three independent cascades: canonical Wnt/ $\beta$-catenin pathway, non-canonical Wnt pathway and Wnt/calcium pathway 9 . Canonical Wnt/ $\beta$-catenin pathway regulates transcription of genes, inhibiting proteolytic degradation of $\beta$-catenin. Lack of Wnt signaling results in a decrease in the cytoplasmic concentration of $\beta$-catenin which forms complexes with E-cadherin ${ }^{10}$. In turn, activation of $\mathrm{Wnt} / \beta$-catenin pathway is reflected by cytoplasmic accumulation of $\beta$-catenin and its translocation to the cell nucleus; here, $\beta$-catenin binds to T-cell factor/lymphoid enhancer factor (TCF/LEF) 
protein, activating transcription of target genes ${ }^{11}$. Therefore, $\beta$-catenin has a dual role in epithelial cells: aside from formation of cell junctions by complexing with E-cadherin, it also acts as a signal transducer within Wnt/ $\beta$-catenin pathway ${ }^{12}$.

A dysfunction of $\mathrm{Wnt} / \beta$-catenin pathway has been implicated in metabolic diseases, congenital defects and oncogenic transformation ${ }^{13,6}$. The latter may result from alterations of canonical Wnt/ $\beta$-catenin pathway and non-canonical Wnt pathway ${ }^{14}$. Approximately $25-47 \%$ of EC patients carry a mutation in $\beta$-catenin gene, ${ }^{15}$ and this protein is considered an early prognostic factor of endometrial carcinogenesis in some cases of atypical hyperplasia ${ }^{16}$.

As transcription factors, special AT-rich binding-sequence proteins 1 and 2 (SATB1/2) interact with nuclear matrix attachment region (MAR), enhancing or suppressing expression of some genes ${ }^{17,18}$. SATB1 contributes to changes in chromatin architecture by organizing chromatin loops centered around the SATB1 core ${ }^{19}$. Overexpression of this protein was shown to promote growth, migration and spread of colon cancer cells ${ }^{20}$.

Defects in SATB1 gene may contribute to development of uterine leiomyomas due to inactivation of $\mathrm{X}$ chromosome. Downregulation of SATB1 was observed in $46 \%$ of leiomyoma specimens, and therefore, this gene was implicated in $X$ chromosome hypomethylation ${ }^{21}$. Similar profile of SATB1 expression was also found in EC specimens. Furthermore, expression of SATB1 was shown to be associated with some clinical characteristics of EC, such as FIGO stage, histological grade and lymph node involvement ${ }^{22}$.

SATB2 is involved in tissue regeneration, promoting survival, migration, angiogenesis and differentiation of cells. While overexpression of SATB2 during the course of regeneration process results in upregulation of other transcriptional proteins, an opposite effect is observed whenever the function of SATB2 is lost. Furthermore, SATB2 was shown to stimulate angiogenesis in mouse skeletal tissue via upregulation of vascular endothelial growth factor (VEGF) ${ }^{23}$.

Previous research documented lack of SATB2 mRNA in both normal uterine tissue and endometrial cancer cells ${ }^{24}$. However, the level of SATB2 in fibroblasts isolated from EC specimens was higher than in normal endometrial fibroblasts. Furthermore, genetic manipulation of SATB2 levels in normal fibroblasts stimulated expression of several genes involved in cell invasion, migration and spread. This implies that SATB2 may be indirectly involved in tumorigenesis, regulating activity of other genes 25 .

During maturation of T-cells, SATB1 interferes with $\mathrm{Wnt} / \beta$-catenin pathway, regulating transcription of some genes. This is $\beta$-catenin which seems to be a link between $W n t / \beta$-catenin signaling pathway and SATB1, as it interacts with the latter protein in cell nucleus. The interaction with $\mathrm{Wnt} / \beta$-catenin pathway was shown to improve DNAbinding activity and transcriptional potential of SATB1, ${ }^{26}$ and was implicated in activation of some oncogenes, promotion of tumorigenesis and metastatic spread of colorectal malignancies ${ }^{27}$.

The aim of this study was to verify if immunoexpression of Wnt3a, $\beta$-catenin, SATB1 and SATB2 in primary tumor is associated with other clinical parameters of EC and may serve as a prognostic factor in patients with this malignancy. 


\section{MATERIALS AND METHODS}

Protocol of the study was approved by the Local Bioethics Committee at the Ludwik Rydygier Collegium Medicum, Nicolaus Copernicus University in Torun, and written informed consent was sought from all the study subjects (decision number KB 332/2007). All patient data were anonymized.

\section{Patients}

The study, conducted between January 2000 and December 2007, included 92 consecutive EC patients, with mean age of $65.1 \pm 9.5$ years (range 37-84 years). EC was diagnosed on the basis of histopathological examination of endometrial specimens obtained by means of curettage. All patients underwent total abdominal hysterectomy with bilateral salpingoophorectomy and pelvic lymph node dissection, performed by experienced gynecological oncologists from the Department of Oncologic Gynecology, Ludwik Rydygier Collegium Medicum in Bydgoszcz, Nicolaus Copernicus University in Torun.

Clinical stage of EC was determined by two independent experienced pathologists on the basis of histopathological examination of surgical specimens, according to the 2009 International Federation of Gynecology and Obstetrics (FIGO) system. The study group included patients with FIGO stages IA ( $n=27)$, IB $(n=18)$, II $(n=14)$, IIIA ( $n=10)$, IIIC $(\mathrm{n}=17)$ and IV $(\mathrm{n}=6)$. Histological grade of the tumors was assessed according to the WHO classification, as G1 (n=7), G2 (n=66) and G3 $(n=19)$. Deep myometrial invasion $(>50 \%)$ was found in 36 patients, lymph node metastases in 23, distant metastases in 6, cervical involvement in 38, and adnexal involvement in 11. Baseline characteristics of the study subjects are presented in Table 1.

Based on their risk profiles, the study subjects were divided into three groups, with low-risk (FIGO IA, G1/G2 with Bokhman type I endometrioid), intermediate-risk (FIGO IA, G3 and IB, G1/G2 with endometrioid type), and high risk (FIGO IB, G3 and FIGO II or higher with endometroid type, and all stages with Bokhman type II - nonendometroid). Subjects from the low-risk group did not receive any adjuvant treatment post-surgery; women from the intermediate-risk group received brachytherapy (VBT) 5 weeks post-surgery, and subjects from the high-risk group were qualified to both teleradiotherapy and VBT. Moreover, 10 patients with non-endometroid EC received adjuvant chemotherapy (carboplatin and paclitaxel).

Patients were followed-up for 60-80 months. Overall survival (OS) was defined as the time from the date of primary treatment to the date of death.

\section{Immunohistochemistry}

Immunohistochemical analyses were conducted on archival formalin-fixed paraffin-embedded EC specimens stored at the Department of Clinical Pathomorphology, Ludwik Rydygier Collegium Medicum, Nicolaus Copernicus University in Torun. Archival paraffin blocks were cut into $3-\mu \mathrm{m}$ sections and immunolabeled as described elsewhere. ${ }^{28}$ Positive controls were prepared in line with The Human Protein Atlas (http://www.proteinatlas.org) ${ }^{29}$ and specifications for the test antibodies; nuclear expression of SATB1 was verified against nongerminal center cells from human tonsils, and expressions of Wnt3a, $\beta$ catenin and SATB2 against glandular cells from human appendix. 
Negative controls were prepared in an analogous way as EC specimens, but with substitution of primary antibodies with a $1 \%$ solution of bovine serum albumin (BSA) diluted in phosphate-buffered saline (PBS).

The analyzed antigens were retrieved with Epitope Retrieval Solution, High-pH (Dako, Glostrup, Denmark) in PT Link (Dako). Then, the activity of endogenous peroxidase and non-specific binding were blocked by 10 -min treatment at room temperature with $3 \% \mathrm{H}_{2} \mathrm{O} 2$ and $5 \%$ BSA, respectively. Subsequently, the slides were incubated with primary antibodies: rabbit polyclonal anti-SATB1 antibody (NBP1-50272 from Novus Biologicals, dilution 1:100, 30-min incubation at $37^{\circ} \mathrm{C}$ ), mouse monoclonal anti-SATB2 antibody (AMAb90635 from Sigma Aldrich, dilution 1:400, 16-h incubation at $4^{\circ} \mathrm{C}$ ), rabbit polyclonal anti-WNT-3A antibody (Cell Signaling 09-162 from Merck Millipore, dilution 1:1000, 30 -min incubation at $37^{\circ} \mathrm{C}$ ) and rabbit monoclonal anti-beta catenin antibody (E247 from Abcam, dilution 1:500, 30-min incubation at $37^{\circ} \mathrm{C}$ ). Antigen-antibody complexes were detected after a 20-min incubation at $37^{\circ} \mathrm{C}$ with EnVisionFLEX-HRP reagent (Dako), on the basis of color reaction with brown chromogen, DAB (3-3'diaminobenzidine). Then, the slides were counterstained with hematoxylin, dehydrated in an increasing ethylene gradient, passed through xylene and sealed.

\section{Evaluation of protein expression}

Immunoexpression of target proteins was analyzed under optic microscope ECLIPSE E400 (Nikon Instruments Europe, Amsterdam, Netherlands) at 20x magnification, using immunoreactive score of Remmele and Stegner ${ }^{30}$ (IRS, from 0 to 12). IRS is calculated as a product of the intensity of color reaction (scored from 0 to 3 , where $0-$ no reaction, 1 - weak reaction, 2 - moderate reaction, 3 - strong reaction) and the proportion of positively-stained cells or specimen area (scored from 0 to 4 , where 0 - no reaction, 1 - less than $10 \%$ of positively-stained cells, $2-10 \%$ to $50 \%$ of positively-stained cells, $3-50 \%$ to $80 \%$ of positively-stained cells, and 4 - more than $80 \%$ of positively-stained cells). IRS scores were determined by two independent pathologists who have been blinded for clinicopathological characteristics of the study subjects. The results $\leq 4$ and $\geq 6$ were interpreted as weak and strong expression of a target protein, respectively.

\section{Statistical analysis}

Statistical analysis of the results was carried out with PQStat software, version 1.4.4.126. The significance of intergroup differences in WNT3A, B-catenin, SATB1 and SATB2 immunoexpression in patients with various clinicopathological characteristics was verified with Kruskal-Wallis test and Mann-Whitney U-test. The differences were considered statistically significant at $\mathrm{p}$-value $<0.05$.

Overall survivals were obtained by Kaplan-Meier method and compared with log-rank test. Moreover, univariate and multivariate Cox regression analyses were conducted to identify significant predictors of OS.

\section{RESULTS}

We have evaluated immunoexpression of WNT3A, B-catenin, SATB1 and SATB2 in primary tumor cells. While the immunoexpression of WNT3A was found in specimens from all EC patients, B-catenin was 
expressed in $97 \%$ of the cases, SATB1 in $87 \%$ and SATB2 in none (Figure $1)$.

Mean IRS values, stratified according to histopathological characteristics of endometrial tumors, are presented in Table 2.

The only histopathological parameter showing a significant association with Wnt3a expression was tumor grade; mean IRS for Wnt3a turned out to be significantly lower in high-grade tumors than in low-grade malignancies $(p=0.038)$. In turn, immunoexpression of $\beta$ catenin varied significantly across FIGO stages and was associated with the presence of lymph node metastases. Mean IRS for $\beta$-catenin in patients with lymph node metastases was significantly lower than in those without $(p=0.028)$. Other analyzed histopathological parameters did not show statistically significant associations with $\beta$-catenin expression (Table 2). Similarly, we found no link between the histopathological characteristics of endometrial tumors and the immunoexpression of SATB1 (Table 2).

All established prognostic factors in EC (FIGO stage, histological grade, lymph node involvement and depth of myometrial invasion) turned out to be significant predictors of 5-year OS on Cox regression analysis.

Analysis of Kaplan-Meier curves demonstrated that the probability of OS increased gradually with WNT3A, B-catenin and SATB1 expression. While immunoexpressions of WNT3A and B-catenin did not exert a significant effect on OS (Figure 2, 3), analysis with log-rank test revealed that higher expression of SATB1 was associated with significantly shorter survival $(p=0.0439)$ (Figure 4$)$.

\section{DISCUSSION}

This study showed that both SATB1 and WNT/ $\beta$-catenin signaling pathway play a role in etiopathogenesis of EC. A growing body of evidence implies that $\mathrm{Wnt} / \beta$-catenin pathway is involved in etiopathogenesis of many malignancies, including female neoplasms, cervical cancer and ovarian cancer, characterized by upregulation of $W_{n t 7 a^{31}}$ and $W n t 5 a,{ }^{32}$ respectively. In the case of EC, the role of $W n t / \beta$ catenin pathway seems to be associated with $\beta$-catenin mutation. Exon-3 mutation in $\beta$-catenin gene and overall $\beta$-catenin expression were shown to be associated with endometroid type of the tumor; in contrast, $\beta$ catenin mutation was not found in non-endometroid $\mathrm{EC}^{15}$. Abnormal expression pattern of $\beta$-catenin was also observed in atypical hyperplasia, which implies that this molecule may be involved at early stages of endometrial carcinogenesis. Indeed, recent evidence suggests that $\beta$-catenin plays a role in the pathogenesis of tumors with Bokhman type 1 features: early-stage, favorable prognosis and endometroid histology ${ }^{16}$. A study in a mouse model demonstrated that altered expression of $\beta$-catenin contributes to endometrial hyperplasia or metaplasia. However, long-term observations showed that although $\beta$ catenin promotes endometrial hyperplasia, its expression is not sufficient for the development of $\mathrm{EC}^{33}$. Indeed, we did not find significant differences in $\beta$-catenin expression in type 1 and type 2 endometrial cancers.

In this study, immunoexpression of $\beta$-catenin in patients without lymph node metastases was significantly higher than in subjects with lymph node involvement, which implies that downregulation of this protein can be considered a predictor of lymphatic spread $(p=0.003)$. In 
line with these findings, immunoexpression of $\beta$-catenin in lymph nodenegative FIGO IIIa tumors turned out to be significantly higher than in node-positive FIGO IIIc malignancies ${ }^{34}$. Although lymph node involvement has unquestioned impact on prognosis in $\mathrm{EC}^{35}$, still little is known about the predictors of lymphatic spread in this maliginancy ${ }^{36}$. Furthermore, there is no consensus if conventional surgical treatment of EC should include elective lymphadenectomy. This justifies research on novel more accurate predictors of lymph node involvement in patients with endometrial malignancies ${ }^{35}$.

Although expression of $\beta$-catenin in EC patients with lymph node metastases generally seems to be weaker, Saegusa et al. found no differences in nuclear and membrane expressions of this protein in nodepositive and node-negative tumors ${ }^{37}$. $\beta$-catenin/E-cadherin complexes are involved in formation of cell junctions and cell-to-cell adhesion. Indeed, previous studies demonstrated that lymphatic spread of various malignancies may be associated with altered expression patterns of Ecadherin ${ }^{38,39}$. Disorders of cell-to-cell adhesion, resulting from malfunction of $\beta$-catenin and E-cadherin, may result in epithelialmesenchymal transition (EMT) and mesenchymal-epithelial reverting transition (MErT). Aside from forming complexes with E-cadherin, $\beta$ catenin regulates activity of Slug and Twist genes; both these genes control E-cadherin expression ${ }^{40}$. The fact that several proteins, such as TGF- $\beta$, EGFR and Snail, are linked to both Wnt/ $\beta$-catenin pathway and EMT/MErT seems to be another argument for the involvement of $\beta$ catenin in tumor spread ${ }^{40}$.

To the best of our knowledge, prognostic value of Wnt3a in EC has not been established thus far. In this study, immunoexpression of Wnt3a was found in all EC specimens. Previously, overexpression of this molecule was reported in breast cancer, gastric cancer, glioma and prostate cancer ${ }^{41}$. According to one theory, Wnt3a may influence tumor progression via EMT-associated proteins, such as E-cadherin, vimentin and $\beta$-catenin ${ }^{42}$. However, in our present study, immunoexpressions of Wnt3a and $\beta$-catenin were associated with different histopathological characteristics of EC; this implies that Wnt3a may interfere with tumor progression via other signaling pathways than those involving $\beta$-catenin.

In colon cancer, Wnt3a seems to promote metastases via interaction with matrix metallopeptidase 9 (MMP-9) and upregulation of vascular endothelial growth factor receptor (VEGFR2) ${ }^{43}$. Indeed, evidence from in vitro studies suggests that Wnt3a promotes angiogenesis due to upregulation of VEGFR2 ${ }^{14}$. However, our findings imply that Wnt3a does not play a role in lymph node and systemic spread of EC, as metastatic and non-metastatic tumors did not differ significantly in terms of its immunoexpressions. In turn, we found statistically significant differences in Wnt3a expressions in low- and high-grade endometrial malignancies. Previously, elevated expression of Wnt3a was found in high-grade gliomas; the same study revealed a correlation between Wnt3a and another activator of Wnt/ $\beta$-catenin pathway, Wnt $1^{44}$. Another study analyzed a link between Wnt10a and Wnt10b expression and clinical characteristics of EC; while Wnt10a was shown to be specific only for histological type of EC, expression of Wnt10b correlated with EC histology, grade, FIGO stage and lymphatic spread ${ }^{38}$. However, other authors did not find significant differences in the immunoexpressions of Wnt1 and Wnt5a in EC and atrophic endometrium ${ }^{45}$. This evidence 
suggests that various types of Wnt proteins may play different roles in tumor progression.

We verified if immunoexpressions of $\beta$-catenin, Wnt3a and SATB1, as well as other clinicopathological factors, may predict 5-year survival in EC. The probability of 5-year survival was lower in patients with latestage EC, deep myometrial invasion, lymph node involvement and high grade of the tumor. In contrast, we did not find a link between OS and the immunoexpressions of Wnt3a and $\beta$-catenin.

However, our hereby presented findings imply that overexpression of SATB1 may be associated with worse prognosis in EC. Somehow similar findings were published by Zhang et al., according to whom expression of SATB1 correlated with worse OS, but also with some clinical characteristics of EC, namely FIGO stage, histological grade and presence of lymph node metastases ${ }^{22}$. However, we did not find significant associations between the immunoexpression of SATB1 and clinicopathological features of EC. Our findings are consistent with the results of previous studies conducted in patients with other malignancies, namely primary bladder cancer ${ }^{46}$, breast cancer ${ }^{47}$ and cervical carcinoma ${ }^{48}$. Overexpression of SATB1 was shown to potentiate cell proliferation and invasiveness, whereas an opposite effect was observed after downregulation of this molecule $27,46,49$.

Upregulation of SATB1 in colon cancer cells was shown to occur secondarily to the activation of $\mathrm{Wnt} / \beta$-catenin pathway; this effect was mediated by a complex of transcription factor 7-like 2 (TCF7L2) and $\beta$ catenin ${ }^{27}$. However, in our study, immunoexpressions of proteins involved in $\mathrm{Wnt} / \beta$-catenin pathway and SATB1 were associated with different clinical characteristics of EC. Furthermore, Kaplan-Meier analysis demonstrated that the probability of OS in EC patients with overexpression of SATB1 was lower than in those without; in contrast, overall survival was not modulated by either Wnt3a or $\beta$-catenin expression. Both SATB1 and Wnt/ $\beta$-catenin pathway contribute to carcinogenesis via EMT pathway ${ }^{40,46}$. In bladder cancer cells, SATB1 modulates EMT pathway, decreasing expression of E-cadherin and stimulating expression of its inhibitors: Snail, Slug and vimentin ${ }^{46}$. SATB1-mediated downregulation of E-cadherin and upregulation of ZEB2, another inducer of EMT pathway, were also documented in renal carcinoma $^{49}$. Downregulation of E-cadherin promotes lymphatic spread and local infiltration of cancer cells due to damage of cell junctions ${ }^{49}$. However, in our present study, lymph node involvement was associated solely with downregulation of $\beta$-catenin, which implies that SATB1 and Wnt/ $\beta$-catenin pathway act independently during endometrial tumorigenesis.

None of the analyzed specimens showed immunoexpression of SATB2. In a previous study, SATB2 and SATB1 exerted opposite effects on c-Myc oncogene in colorectal cancer cells, contributing to its suppression and upregulation, respectively ${ }^{50}$. In line with these findings, downregulation of SATB2 was shown to correlate with less favorable clinicopathological profile and worse survival in clear cell renal carcinoma $^{51}$ and esophageal squamous cell carcinoma ${ }^{52}$. However, quite contradictory results were obtained in an in vitro model of endometrial cancer fibroblasts, whereby upregulation of SATB2 resulted in greater cellular invasiveness and migratory potential ${ }^{25}$. SATB2 may be regulated by a multitude of miRNAs and pathways, which further highlights the complexity of SATB2's involvement in cancer ${ }^{53}$.These discrepancies 
justify further research on the role of SATB2 in endometrial tumorigenesis.

In conclusion, the hereby presented findings imply that both $\mathrm{Wnt} / \beta$ catenin signaling pathway and SATB1 contribute to progression of EC. Although both $\beta$-catenin and Wnt3a are involved in the same $W n t / \beta$ catenin pathway, their immunoexpressions correlate with different clinicopathological characteristics of EC. Downregulation of $\beta$-catenin may predispose to lymphatic spread of EC. In turn, downregulation of Wnt3a seems to be characteristic for high-grade tumors, but probably does not play a role in formation of lymph node metastases. While the expression of SATB2 in EC is likely inhibited, overexpression of another transcription factor, SATB1 seems to be associated with lower probability of survival.

Acknowledgments: The authors would like to acknowledge Prof. Marek Grabiec for his keen interest, inspiring guidance, and consist encouragement.

\section{REFERENCES:}

1. Justus C, Sanderlin E, Yang L. Molecular Connections between Cancer Cell Metabolism and the Tumor Microenvironment. Int J Mol Sci. 2015;16(5):11055-11086. doi:10.3390/ijms160511055.

2. Ferlay J, Steliarova-Foucher E, Lortet-Tieulent J, et al. Cancer incidence and mortality patterns in Europe: estimates for 40 countries in 2012. Eur J Cancer. 2013;49(6):1374-1403. doi:10.1016/j.ejca.2012.12.027.

3. Amant F, Moerman P, Neven P, Timmerman D, Van Limbergen E, Vergote I. Endometrial cancer. Lancet (London, England). 366(9484):491-505. doi:10.1016/S0140-6736(05)67063-8.

4. Wend P, Holland JD, Ziebold U, Birchmeier W. Wnt signaling in stem and cancer stem cells. Semin Cell Dev Biol. 2010;21(8):855-863. doi:10.1016/j.semcdb.2010.09.004.

5. van Amerongen R, Nusse R. Towards an integrated view of Wnt signaling in development. Development. 2009;136(19):3205-3214. doi:10.1242/dev.033910.

6. Clevers H, Nusse R. Wnt/ $\beta$-catenin signaling and disease. Cell. 2012;149(6):1192-1205. doi:10.1016/j.cell.2012.05.012.

7. Hausmann G, Bänziger C, Basler K. Helping Wingless take flight: how WNT proteins are secreted. Nat Rev Mol Cell Biol. 2007;8(4):331-336. doi:10.1038/nrm2141.

8. Willert K, Brown JD, Danenberg E, et al. Wnt proteins are lipid-modified and can act as stem cell growth factors. Nature. 2003;423(6938):448-452. doi:10.1038/nature01611.

9. Nusse R, Varmus H. Three decades of Wnts: a personal perspective on how a scientific field developed. EMBO J. 2012;31(12):2670-2684. doi:10.1038/emboj.2012.146.

10. Reya T, Clevers H. Wnt signalling in stem cells and cancer. Nature. 2005;434(7035):843-850. doi:10.1038/nature03319.

11. Wallmen B, Schrempp M, Hecht A. Intrinsic properties of Tcf1 and Tcf4 splice variants determine cell-typespecific Wnt/ $\beta$-catenin target gene expression. Nucleic Acids Res. 2012;40(19):9455-9469. doi:10.1093/nar/gks690.

12. Ghahhari NM, Babashah S. Interplay between microRNAs and WNT/ $\beta$-catenin signalling pathway regulates epithelial-mesenchymal transition in cancer. Eur J Cancer. 2015;51(12):1638-1649. doi:10.1016/j.ejca.2015.04.021.

13. Hanahan D, Weinberg RA. The hallmarks of cancer. Cell. 2000;100(1):57-70. http://www.ncbi.nlm.nih.gov/pubmed/10647931. Accessed April 9, 2016.

14. Qi L, Song W, Liu Z, Zhao X, Cao W, Sun B. Wnt3a Promotes the Vasculogenic Mimicry Formation of Colon Cancer via Wnt/ $\beta$-Catenin Signaling. Int J Mol Sci. 2015;16(8):18564-18579. doi:10.3390/ijms160818564.

15. Moreno-Bueno G, Hardisson D, Sánchez C, et al. Abnormalities of the APC/beta-catenin pathway in endometrial cancer. Oncogene. 2002;21(52):7981-7990. doi:10.1038/sj.onc.1205924.

16. van der Zee M, Jia Y, Wang Y, et al. Alterations in Wnt- $\beta$-catenin and Pten signalling play distinct roles in endometrial cancer initiation and progression. J Pathol. 2013;230(1):48-58. doi:10.1002/path.4160.

17. Zhao X, Qu Z, Tickner J, Xu J, Dai K, Zhang X. The role of SATB2 in skeletogenesis and human disease. Cytokine Growth Factor Rev. 2014;25(1):35-44. doi:10.1016/j.cytogfr.2013.12.010.

18. Nakagomi K, Kohwi Y, Dickinson LA, Kohwi-Shigematsu T. A novel DNA-binding motif in the nuclear matrix attachment DNA-binding protein SATB1. Mol Cell Biol. 1994;14(3):1852-1860. http://www.ncbi.nlm.nih.gov/pubmed/8114718. Accessed April 18, 2016.

19. Kohwi-Shigematsu T, Kohwi Y, Takahashi K, et al. SATB1-mediated functional packaging of chromatin into 
loops. Methods. 2012;58(3):243-254. doi:10.1016/j.ymeth.2012.06.019.

20. Fang X-F, Hou Z-B, Dai X-Z, et al. Special AT-rich sequence-binding protein 1 promotes cell growth and metastasis in colorectal cancer. World J Gastroenterol. 2013;19(15):2331-2339. doi:10.3748/wjg.v19.i15.2331.

21. Sato S, Maekawa R, Yamagata Y, et al. Potential mechanisms of aberrant DNA hypomethylation on the $x$ chromosome in uterine leiomyomas. $J$ Reprod Dev. 2014;60(1):47-54. http://www.ncbi.nlm.nih.gov/pubmed/24291816. Accessed May 7, 2016.

22. Zhang Y, Wang L, Liu Y, et al. Overexpression of special AT-rich sequence-binding protein 1 in endometrial cancer: a clinicopathologic study. Int J Gynecol Cancer. 2015;25(1):4-11. doi:10.1097/IGC.0000000000000314.

23. Zhang J, Tu Q, Grosschedl R, et al. Roles of SATB2 in osteogenic differentiation and bone regeneration. Tissue Eng Part A. 2011;17(13-14):1767-1776. doi:10.1089/ten.TEA.2010.0503.

24. Magnusson K, de Wit M, Brennan DJ, et al. SATB2 in combination with cytokeratin 20 identifies over $95 \%$ of all colorectal carcinomas. Am J Surg Pathol. 2011;35(7):937-948. doi:10.1097/PAS.0b013e31821c3dae.

25. Aprelikova $\mathrm{O}, \mathrm{Yu}$ X, Palla J, et al. The role of miR-31 and its target gene SATB2 in cancer-associated fibroblasts. Cell Cycle. 2010;9(21):4387-4398. http://www.ncbi.nlm.nih.gov/pubmed/20980827. Accessed April 9, 2016.

26. Notani D, Gottimukkala KP, Jayani RS, et al. Global regulator SATB1 recruits beta-catenin and regulates $\mathrm{T}(\mathrm{H}) 2$ differentiation in Wnt-dependent manner. PLoS Biol. 2010;8(1):e1000296. doi:10.1371/journal.pbio.1000296.

27. Mir R, Pradhan SJ, Patil P, Mulherkar R, Galande S. Wnt/ $\beta$-catenin signaling regulated SATB1 promotes colorectal cancer tumorigenesis and progression. Oncogene. 2016;35(13):1679-1691. doi:10.1038/onc.2015.232.

28. Bodnar M, Szylberg L, Kazmierczak W, Marszalek A. Tumor progression driven by pathways activating matrix metalloproteinases and their inhibitors. J Oral Pathol Med. 2014;44(6):437-443. doi:10.1111/jop.12270.

29. Uhlen M, Oksvold P, Fagerberg L, et al. Towards a knowledge-based Human Protein Atlas. Nat Biotechnol. 2010;28(12):1248-1250. doi:10.1038/nbt1210-1248.

30. Remmele W, Stegner HE. [Recommendation for uniform definition of an immunoreactive score (IRS) for immunohistochemical estrogen receptor detection (ER-ICA) in breast cancer tissue]. Pathologe. 1987;8(3):138140.

31. Ramos-Solano M, Meza-Canales ID, Torres-Reyes LA, et al. Expression of WNT genes in cervical cancerderived cells: Implication of WNT7A in cell proliferation and migration. Exp Cell Res. 2015;335(1):39-50. doi:10.1016/j.yexcr.2015.05.001.

32. Burkhalter RJ, Symowicz J, Hudson LG, Gottardi CJ, Stack MS. Integrin regulation of beta-catenin signaling in ovarian carcinoma. J Biol Chem. 2011;286(26):23467-23475. doi:10.1074/jbc.M110.199539.

33. Jeong J-W, Lee HS, Franco HL, et al. beta-catenin mediates glandular formation and dysregulation of betacatenin induces hyperplasia formation in the murine uterus. Oncogene. 2009;28(1):31-40. doi:10.1038/onc.2008.363.

34. Zaino RJ. FIGO staging of endometrial adenocarcinoma: a critical review and proposal. Int J Gynecol Pathol. 2009;28(1):1-9. doi:10.1097/PGP.0b013e3181846c6d.

35. Werner HMJ, Trovik J, Marcickiewicz J, et al. Revision of FIGO surgical staging in 2009 for endometrial cancer validates to improve risk stratification. Gynecol Oncol. 2012;125(1):103-108. doi:10.1016/j.ygyno.2011.11.008.

36. Son J-H, Kong T-W, Kim SH, et al. Prediction of lymph node metastasis in patients with apparent early endometrial cancer. Obstet Gynecol Sci. 2015;58(5):385-390. doi:10.5468/ogs.2015.58.5.385.

37. Saegusa M, Hashimura M, Yoshida T, Okayasu I. beta- Catenin mutations and aberrant nuclear expression during endometrial tumorigenesis. Br J Cancer. 2001;84(2):209-217. doi:10.1054/bjoc.2000.1581.

38. Chen L, Jian W, Lu L, Zheng L, Yu Z, Zhou D. Elevated expression of E-cadherin in primary breast cancer and its corresponding metastatic lymph node. Int J Clin Exp Med. 2015;8(7):11752-11758. http://www.ncbi.nlm.nih.gov/pubmed/26380015. Accessed April 9, 2016.

39. Jiang Y, Liao L, Shrestha C, et al. Reduced expression of E-cadherin and p120-catenin and elevated expression of PLC $-\gamma 1$ and PIKE are associated with aggressiveness of oral squamous cell carcinoma. Int $J$ Clin Exp Pathol. 2015;8(8):9042-9051. http://www.ncbi.nlm.nih.gov/pubmed/26464646. Accessed April 9, 2016.

40. $\mathrm{Fu} \mathrm{Y}$, Zheng $\mathrm{S}, \mathrm{An} \mathrm{N}$, et al. $\beta$-catenin as a potential key target for tumor suppression. Int $J$ cancer. 2011;129(7):1541-1551. doi:10.1002/ijc.26102.

41. He S, Lu Y, Liu X, et al. Wnt3a: functions and implications in cancer. Chin J Cancer. 2015;34(12):554-562. doi:10.1186/s40880-015-0052-4.

42. Qi L, Sun B, Liu Z, Cheng R, Li Y, Zhao X. Wnt3a expression is associated with epithelial-mesenchymal transition and promotes colon cancer progression. J Exp Clin Cancer Res. 2014;33:107. doi:10.1186/s13046-0140107-4.

43. Lee MA, Park J-H, Rhyu SY, Oh S-T, Kang W-K, Kim H-N. Wnt3a expression is associated with MMP-9 expression in primary tumor and metastatic site in recurrent or stage IV colorectal cancer. BMC Cancer. 2014;14:125. doi:10.1186/1471-2407-14-125. 
44. Kaur N, Chettiar S, Rathod S, et al. Wnt3a mediated activation of Wnt//-catenin signaling promotes tumor progression in glioblastoma. Mol Cell Neurosci. 2013;54:44-57. doi:10.1016/j.mcn.2013.01.001.

45. Menezes M de PN, Oshima CTF, Filho LB, et al. Canonical and noncanonical Wnt pathways: a comparison between endometrial cancer type I and atrophic endometrium in Brazil. São Paulo Med J = Rev Paul Med. 2011;129(5):320-324. http://www.ncbi.nlm.nih.gov/pubmed/22069131. Accessed April 9, 2016.

46. Wan F, Cheng C, Wang Z, et al. SATB1 overexpression regulates the development and progression in bladder cancer through EMT. PLoS One. 2015;10(2):e0117518. doi:10.1371/journal.pone.0117518.

47. Liu X, Zheng Y, Qiao C, et al. Expression of SATB1 and HER2 in breast cancer and the correlations with clinicopathologic characteristics. Diagn Pathol. 2015;10:50. doi:10.1186/s13000-015-0282-4.

48. Wang S, Wang L, Zhang Y, et al. Special AT-rich sequence-binding protein 1: a novel biomarker predicting cervical squamous cell carcinoma prognosis and lymph node metastasis. Jpn J Clin Oncol. 2015;45(9):812-818. doi:10.1093/jjco/hyv093.

49. Cheng C, Wan F, Liu L, et al. Overexpression of SATB1 is associated with biologic behavior in human renal cell carcinoma. PLoS One. 2014;9(5):e97406. doi:10.1371/journal.pone.0097406.

50. Mansour MA, Hyodo T, Akter KA, et al. SATB1 and SATB2 play opposing roles in c-Myc expression and progression of colorectal cancer. Oncotarget. 2016;7(4):4993-5006. doi:10.18632/oncotarget.6651.

51. Guo C, Xiong D, Yao X, et al. Decreased SATB2 expression is associated with metastasis and poor prognosis in human clear cell renal cell carcinoma. Int J Clin Exp Pathol. 2015;8(4):3710-3718. http://www.ncbi.nlm.nih.gov/pubmed/26097552. Accessed May 15, 2016.

52. Geng G-J, Li N, Mi Y-J, et al. Prognostic value of SATB2 expression in patients with esophageal squamous cell carcinoma. Int J Clin Exp Pathol. 2015;8(1):423-431. http://www.ncbi.nlm.nih.gov/pubmed/25755730. Accessed May 15, 2016.

53. Qiao Yi Chen, Thomas Des Marais, Max Costa Deregulation of SATB2 in carcinogenesis with emphasis on miRNA-mediated control. Carcinogenesis. 2019 May; 40(3): 393-402. doi: 10.1093/carcin/bgz020

Table 1. Baseline characteristics of endometrial cancer patients.

\begin{tabular}{|c|c|c|}
\hline & & N (\%) \\
\hline \multirow{6}{*}{ FIGO stage } & $\mathrm{IA}$ & $27(29.35 \%)$ \\
\hline & $\mathrm{IB}$ & $18(19.57 \%)$ \\
\hline & $\|$ & $14(15.22 \%)$ \\
\hline & IIIA & $10(10.87 \%)$ \\
\hline & $\mathrm{IIIC}$ & $17(18.48 \%)$ \\
\hline & IVB & $6(6.52 \%)$ \\
\hline \multirow{3}{*}{ Grade } & G1 & $7(7.61 \%)$ \\
\hline & G2 & $66(71.74 \%)$ \\
\hline & G3 & $19(20.65 \%)$ \\
\hline \multirow{2}{*}{ Bokhman subtype } & Endometrioid (I) & $70(76.09 \%)$ \\
\hline & Non-endometrioid (II) & $22(23.91 \%)$ \\
\hline \multirow{2}{*}{ Lymph node metastases (N) } & Absent NO & $69(75 \%)$ \\
\hline & Present N1 & $23(25 \%)$ \\
\hline \multirow{2}{*}{ Distant metastases (M) } & Absent $M 0$ & $86(93.48 \%)$ \\
\hline & Present Ml & $6(6.52 \%)$ \\
\hline \multirow{2}{*}{ Myometrial invasion } & $<50 \%$ & $56(60.87 \%)$ \\
\hline & $\geq 50 \%$ & $36(39.13 \%)$ \\
\hline \multirow{2}{*}{ Cervical involvement } & Absent & $54(58.70 \%)$ \\
\hline & Present & $38(41.30 \%)$ \\
\hline \multirow{2}{*}{ Adnexal involvement } & Absent & $81(88.04 \%)$ \\
\hline & Present & $11(11.96 \%)$ \\
\hline
\end{tabular}

Table 2. Immunoexpressions of Wnt3a, $\beta$-catenin and SATB1 in surgical endometrial cancer specimens. Patients were divided into low-, intermediate- and high-risk groups based on FIGO stage, histological grade (G1, G2 and G3), histological type according to Bokhman (type 1 - endometrioid, type 2 - non-endometrioid), absence (N0) or presence of lymph node metastases (N1), absence (M0) or presence (M1) of distant metastases, depth of myometrial invasion (less than $50 \%$ or more than $50 \%$ ), absence $(=0)$ or presence $(=1)$ of cervical involvement, and absence $(=0)$ or presence $(=1)$ of. 


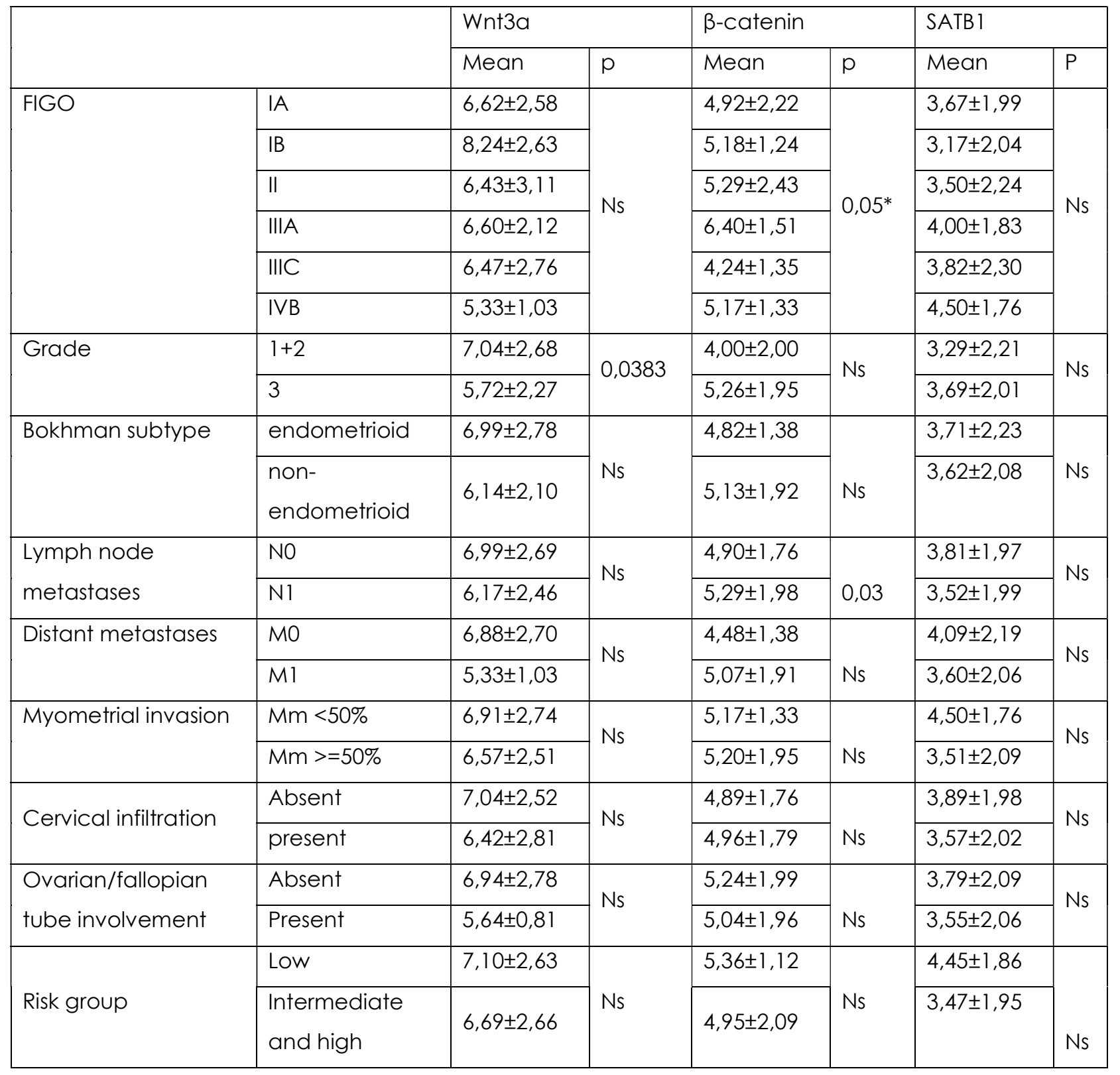

ovarian and/or fallopian tube infiltration. 
*significant difference between FIGO stages IIIA and IIIC

$\mathrm{N}=$ lymph node metastases, $\mathrm{M}=$ distant metastases, $\mathrm{Mm}>50 \%$ = depth of myometrial invasion $>50 \%$, Ns=non-significant

Table 3. Results of Cox regression analysis illustrating role of $\beta$-catenin, Wnt3a, FIGO stage III and IV, grade III, type 2 of endometrial cancer according to Bokhman, lymph node involvement and $>50 \%$ depth of myometrial invasion as predictors of 5-year overall survival.

\begin{tabular}{ccc}
\hline & & \\
\cline { 2 - 3 } & HR [95\%Cl] & P \\
\hline B-catenin & 0,96 \\
\hline Wnt3a & $1,00[0,82-1,21]$ & 0,29 \\
\hline SATB 1 & $0,92[0,79-1,07]$ & $<0,01$ \\
\hline FIGO (III+IV) & 0,01 \\
\hline G (3) & $0,33[0,16-0,70]$ & 0,71 \\
\hline Type 2 by Bokhman & $0,34[0,16-0,74]$ & $<0,01$ \\
\hline Mm $>50 \%$ & $0,86[0,38-1,94]$ & $<0,01$ \\
\hline
\end{tabular}

$\mathrm{HR}=$ hazard ratio, $\mathrm{CI}=$ confidence interval, $\mathrm{N}=$ lymph node metastases, $\mathrm{Mm}>50 \%=$ more than $50 \%$ depth of myometrial invasion. 


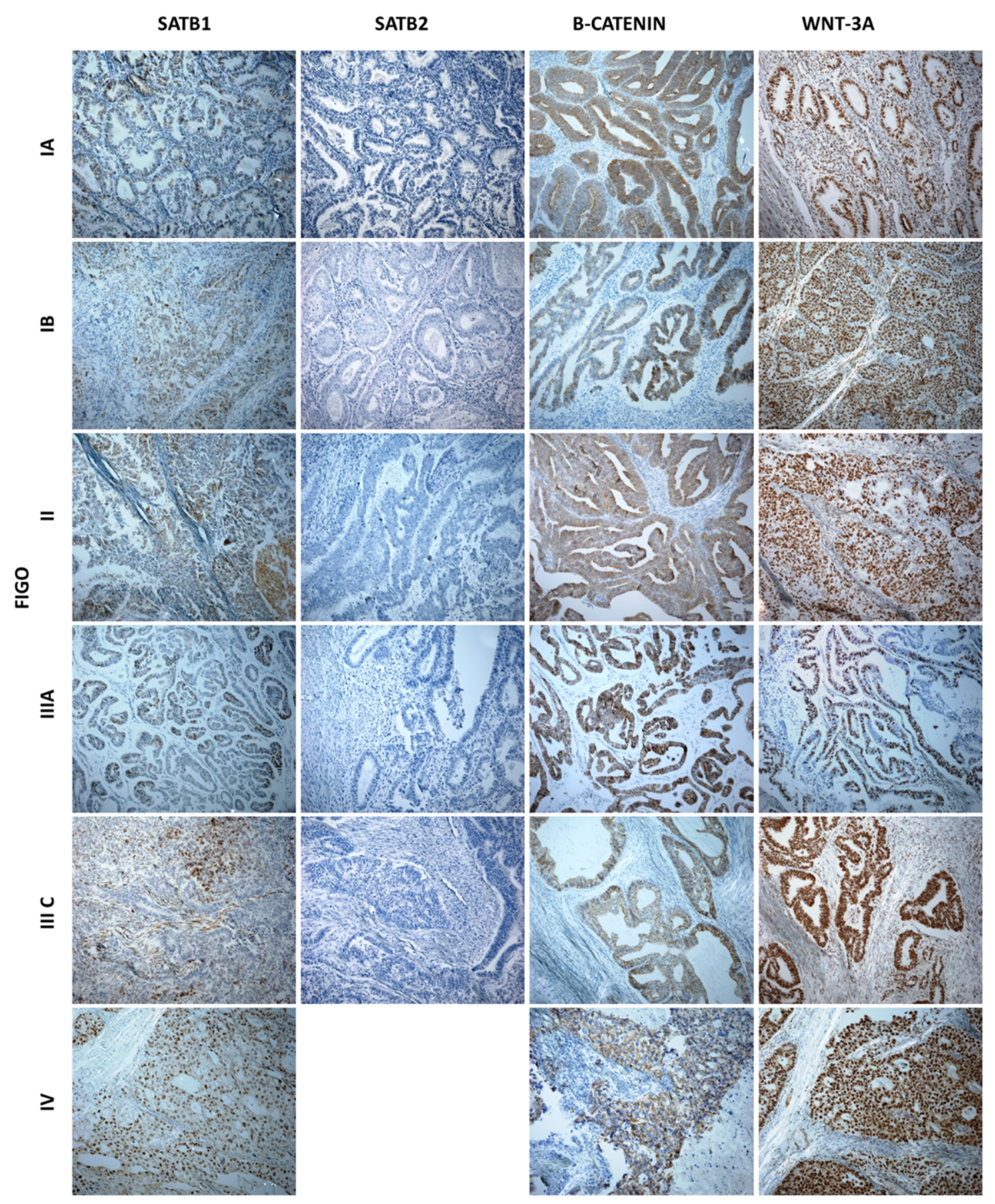

Figure 1. Representative immunohistochemical microphotographs (brown color - positive reaction) presenting the nuclear STAB1, nuclear SATB2, membrane B-CATENIN and nuclear WNT-3A expression in endometrial cancer according to FIGO classification (IA, IB, II, IIIA, IIIC, IV). Nucleus counterstained with hematoxylin. Primary objective magnification 10x. 


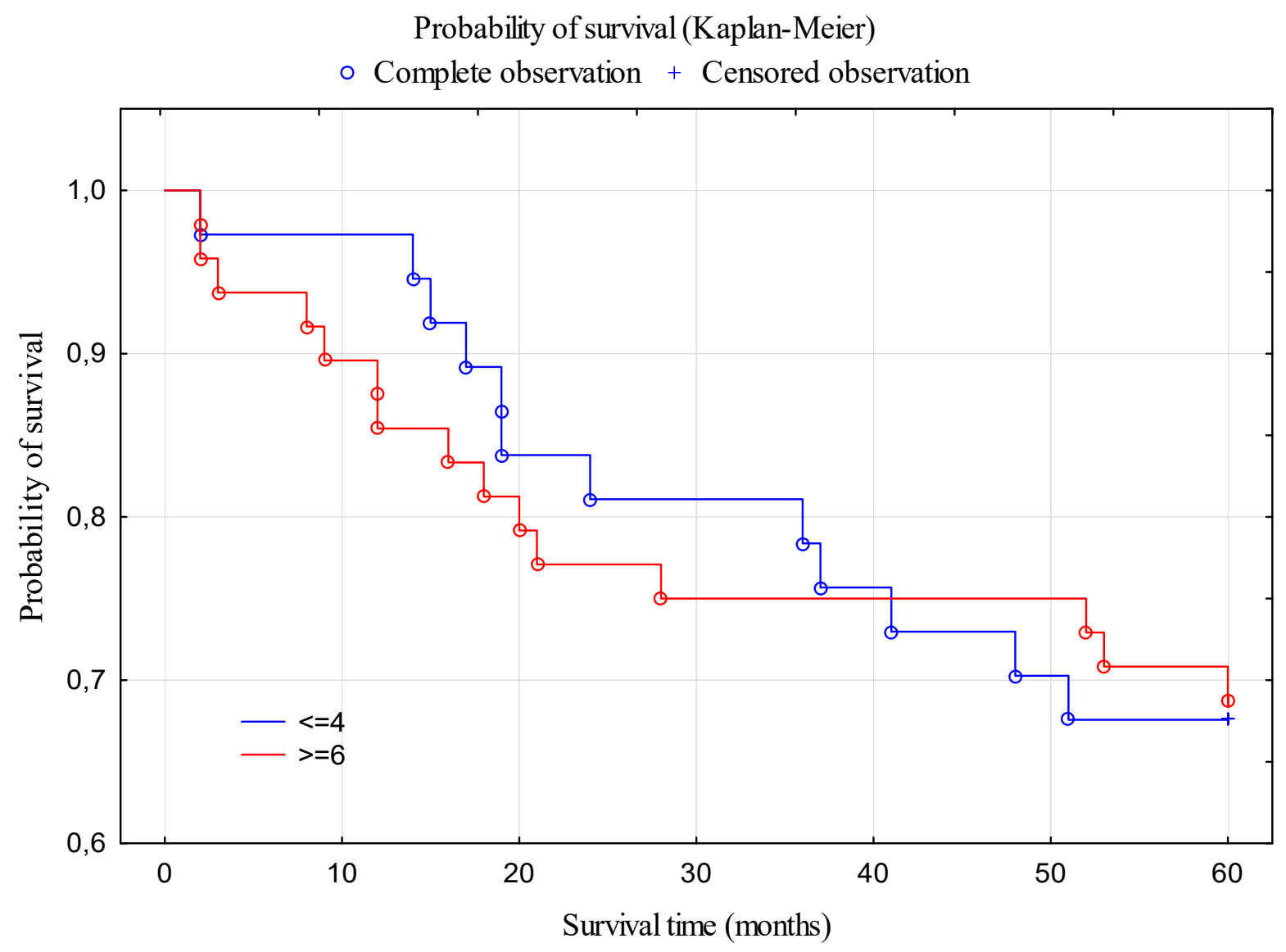

Figure 2. Kaplan-Meier curves illustrating a relationship between immunoexpression of WNT3A and overall survival in endometrial cancer patients. 


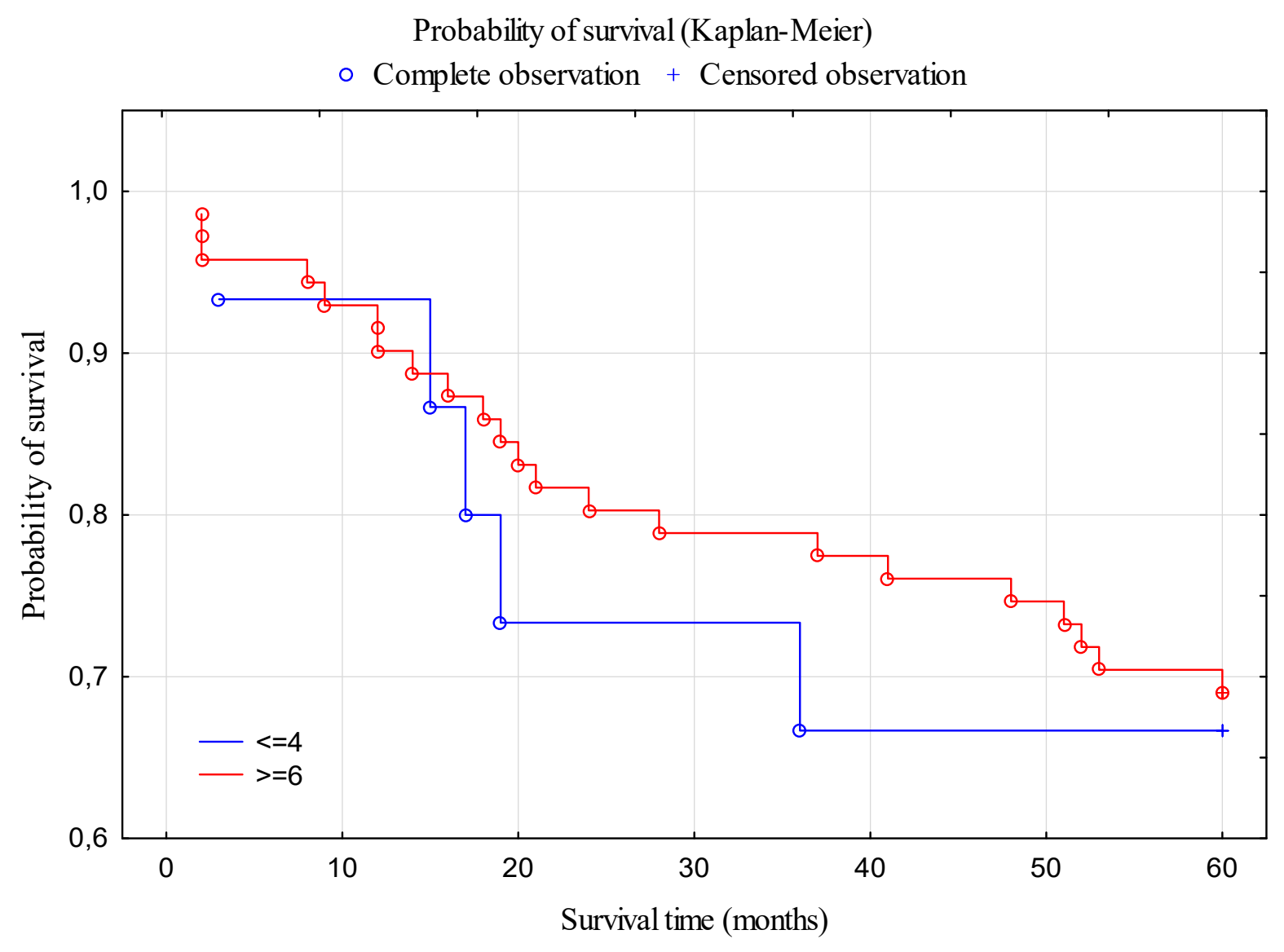

Figure 3. Kaplan-Meier curves illustrating a relationship between immunoexpression of $\beta$ catenin and overall survival in endometrial cancer patients. 


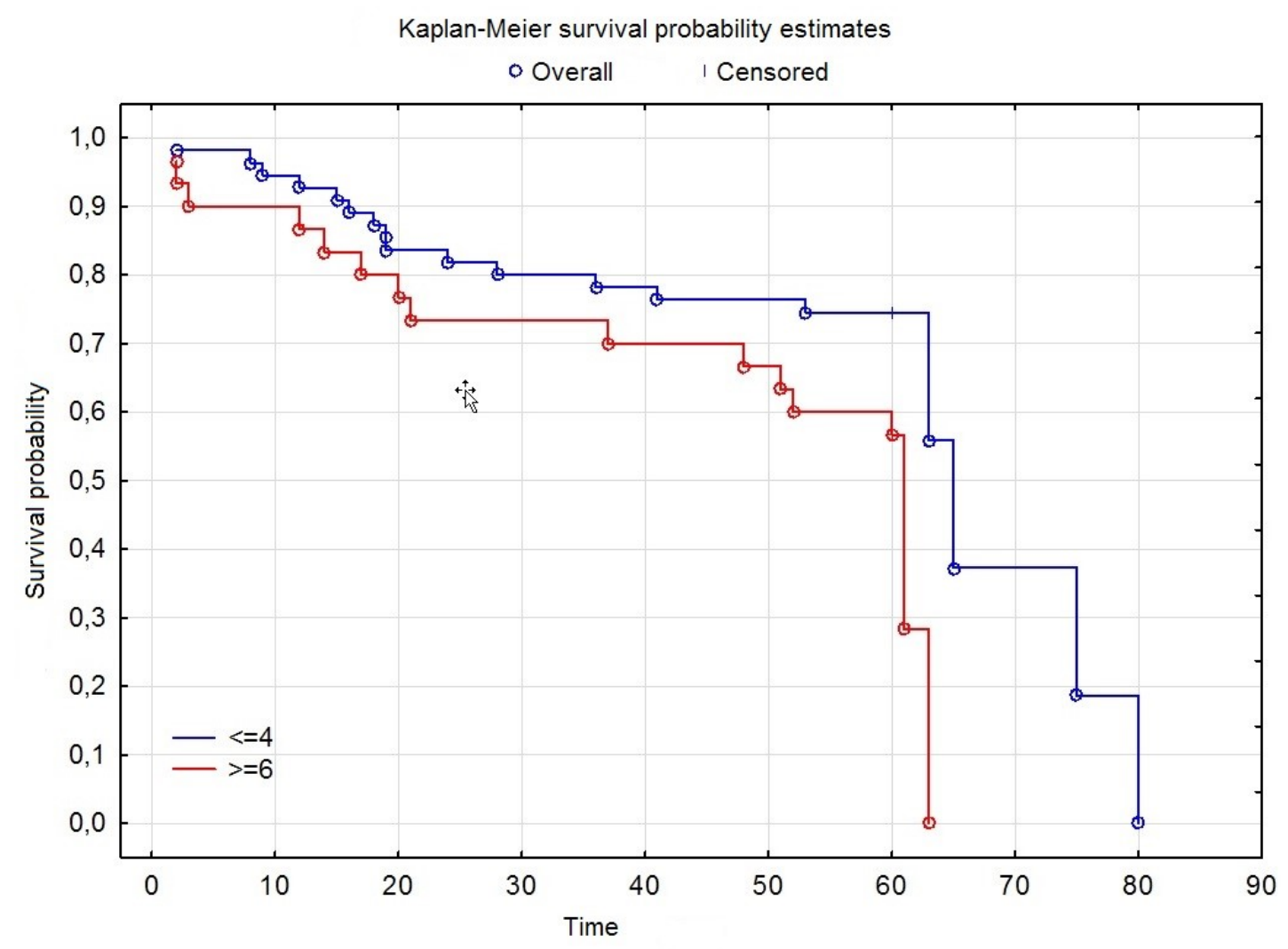

Figure 4. Kaplan-Meier curves illustrating a relationship between immunoexpression of SATBI and overall survival in endometrial cancer patients. 